\title{
Efficacy of Probiotics for Prevention of Small Intestinal Bacterial Overgrowth (SIBO) Recurrence among Patients with Irritable Bowel Syndrome (IBS)
}

\author{
Abdol-Rahim Masjedizadeh ${ }^{1,2}$, Pezhman Alavinejad ${ }^{1,2}$, \\ Sam Shahinzadeh ${ }^{\mathbf{1}, 2}$ \\ ${ }^{1}$ Alimentary Tract Research Center, Ahvaz Jundishapur \\ University of Medical Sciences, Iran. \\ ${ }^{2}$ Department of Internal Medicine, Ahvaz, Jundishapur University of Medical Sciences, \\ Iran.
}

Corresponding Author Dr Pezhman Alavinejad

Mobile: 00989161115880

E mail: pezhmanalavinejad@g mail.com

Key words: Irritable Bowel Syndrome, Small Intestinal Bacterial Overgrowth, Probiotic
Introduction and study aim: IBS is the most common functional disorder of gastrointestinal (GI) tract. It is shown that intestinal microbiota plays an important role in the pathogenesis of Irritable Bowel Syndrome (IBS). Recent studies have been shown that probiotics could be effective in the improvement of IBS symptoms by changing gut normal microflora. In this study we investigated the probiotics' efficacy in prevention of small intestinal bacterial overgrowth (SIBO) recurrence among patients with IBS.

Patients and Methods: In a double blind clinical trial, patients with IBS and SIBO who were diagnosed based on positive Hydrogen breath test, initially received antibiotics for a period of 10 days and then randomly divided into 2 groups (Mutaflor probiotic and placebo) after confirmation of negative Hydrogen breath test. After 3 months treatment, breath tests were repeated and frequency of SIBO recurrence was compared between 2 groups.

Results: Among 172 patients with IBS and SIBO, 159 cases included the study and after antibiotic treatment and negative breath tests, eventually 156 patients were analyzed. Both groups had similar demographic characters such as age, sex, IBS type and PPI consumption. Frequency of SIBO recurrence were significantly lower in the probiotic group than the placebo group $(\mathrm{P}=0.033)$. Frequency rate of constipation and mixed type of IBS were almost similar in both groups $(\mathrm{P}>0.05)$ and only there was a significant difference in SIBO frequency between 2 groups as diarrhea dominant IBS $(\mathrm{P}<0.05)$

Conclusion: Our study showed that Mutaflor probiotic has beneficial effects on reduction of SIBO recurrence among IBS patients and could be successfully used for treatment of these patients.

\section{INTRODUCTION}

Irritable bowel syndrome (IBS) is the most common gastrointestinal (GI) disorder which is diagnosed with chronic abdominal pain, bloating and changes in bowel movements in the absence of any physiological disorder. It has a negative impact on the quality of life, daily activity, and work and leisure time of the patients $[1,2]$. In the recent years, there have been increasing interests in supporting the effect of introducing changes in the combination of intestinal bacteria on IBS. Some of the studies found the correlation between small intestine bacteria overgrowth (SIBO) and IBS by using a respiratory test $[\mathbf{1}, \mathbf{3}, \mathbf{4}]$.

On the other hand, the occurrence of SIBO is also high after the course of antibiotic treatment (up to $40 \%$ during nine months after treatment) and the onset of SIBO in patients with IBS can lead to regular use of antibiotics. Hence, this condition can result in increase of the symptoms and financial expenses, and a decrease in the quality of life with several other side effects $[\mathbf{5 , 6}]$.

Masjedizadeh et al., Afro-Egypt J Infect Endem Dis 2020;10(3):279-286 https://aeji.journals.ekb.eg/

http://mis.zu.edu.eg/ajied/home.aspx 
Recent studies concluded that treatment of gastrointestinal tract with probiotic bacteria could be effective in improvement of the symptoms. This recommendation is based on the fact that probiotics can improve the normal balance of intestinal flora $[7,1]$. Based on these findings, many research studies have been designed to assess the impact of different probiotics on the improvement of gastrointestinal symptoms among IBS patients. Some of these studies have concluded that probiotics are beneficial, and the other studies did not support this statement $[\mathbf{1}, \mathbf{3}, \mathbf{8}, \mathbf{9}]$.

It should be mentioned that all of the studies which have been conducted so far regarding the control of IBS with probiotics, have assessed the improvement of a gastrointestinal symptoms as a result of different probiotics consumption. Therefore, our main objective of this study is to evaluate the condition of gastrointestinal flora which is an objective variable instead of measuring gastrointestinal symptoms as a subjective variable. Our goal is to understand whether probiotics consumption can prevent the further onset of SIBO in the small intestine which is usually associated with IBS symptoms in most studies.

\section{PATIENTS AND METHODS}

In this randomized clinical trial, after obtaining ethical committee approval from Ahvaz Jundishapur University of Medical Sciences (AJUMS), during a six months period, all the patients with IBS were selected among the referred patients from the gastroenterology clinics of Imam Khomeini Hospital, Golestan Hospital and private gastroenterology clinics in Ahvaz city. IBS patients were selected by academic gastroenterologists based on the ROME IV criteria [23]. Patients with a history of any major disease such as IBD, coeliac disease and/or lack of enzyme disorders were excluded from the study.

The following tests were performed for all of the participants at the beginning of the study:

- Full medical history, biochemical profile, CRP, ESR, CBC, feces pathology for negative blood parasites, egg parasites, antibodies and anti-transglutaminase antibody (Anti TTG).

- X-ray from abdominal cavity.
- Colonoscopy in case of alarming symptoms such as fever, gastrointestinal bleeding, weight loss, anemia or abdominal mass.

The exclusion criteria of the study including:

- Patients age under 18 and over 70

- Sensitivity to antibiotics

- Pregnancy or breastfeeding

- History of major abdominal surgery

- Having major disease such as cancer, liver failure, kidney failure, hypothyroidism, diabetes and Scleroderma.

- Active organic gastrointestinal tract disorder.

- Family history of colorectal carcinoma or IBD.

- current treatment with immunosuppressive agents or high dose of steroids.

- Treatment with antibiotics in the past two months prior to the study.

- Using probiotics more than two weeks in the past six months.

- Positive hydrogen breath test after the course of antibiotics.

Firstly, all the participants were tested for Hydrogen Breathing Test (HBT) with MicroH2 and Hider machine made in Micro Medical LTD Co. in the United Kingdom. In order to diagnose SIBO all of the patients were fasted for 12 hours prior to the test without doing exercise and smoking during this period. To eliminate the effects of oral bacteria floras on the outcomes of the study, all the patients used $30 \mathrm{~mL}$ of Chlor hexidine $1 \%$ mouthwash one hour before the test. First, one base specimen was taken from the participants, then patients were given $1 \mathrm{~g}$ of glucose per each kilograms of bodyweight dissolved in $150 \mathrm{~mL}$ of water, then hydrogen in expiration were measured every 30 minutes up to 4 times. If the hydrogen level was more than 12 ppm, the test was considered positive.

After that, 172 patients with SIBO were treated with Co-amoxiclav (Amoxicilline/clavulanate) $(1300 \mathrm{mg} /$ day) and metronidazole $(1 \mathrm{~g} /$ day $)$ for 10 days. After the course of treatment, the hydrogen breathing test (HBT) was repeated to confirm the cure of SIBO. The patients with negative hydrogen breathing test were selected for further study while patients with positive hydrogen breathing test were excluded from this 
research. Patients with a negative HBT were randomly divided into two groups (Random number selection table). Probiotic Mutaflor hard capsule was given to one group of patients and placebo was given to the other group. Each probiotic capsule contains E. coli strain Nissle 1917 (8.74 - 43.68mg) with $2.5-25 \times 10 * 9$ viable cells made by ARDEYPHARM-GmbH in Germany, and the placebo were resembled with probiotic capsules by Pharmacy department of AJUMS.

After three months, the hydrogen breathing test was repeated to compare the outcomes.

All demographic data obtained from the participant were analyzed by SPSS V19.0 Software. Statistical test such as Pearson's Chisquare test (X2) and Fisher's exact test used in order to compare the onset of SIBO in placebo group and probiotic group. In the case of $\mathrm{P}$ value $<0.05$, the test considered as significant. Other descriptive tests such as frequency distribution tables and charts were also used to describe the findings.

\section{RESULTS}

In this randomized clinical trial, 172 patients with IBS according to ROME IV criteria included. The average age of the patients was $36.40 \pm 9.58 y$ and the age range was $20-58$ years old. $36.6 \%$ of the patients were male with average age of $36.66 \pm 9.27$ and $63.4 \%$ were female with average of $36.25 \pm 9.79(\mathrm{P}=78.0)$.

The most common complaints were abdominal pain in $87 \%$ of the patients and bloating in $79 \%$ of the patients. $48.3 \%$ of the patients had constipation dominant IBS (IBS-C), 30.8\% suffered with diarrhea type (IBS-D) and $20.9 \%$ were mixed type (IBS-M). There was no statistically significant difference between male and female in the terms of the types of IBS $(\mathrm{P}=0.62)$.

After antibiotic treatment and repeating HBT, 13 patients $(7.55 \%)$ were excluded from this study due to positive breath test results. The remaining 159 patients with negative HBT were randomly divided into 2 groups of probiotics (79 patients) and placebo (80 patients) (figure 1). There was not a significant difference between the average age, gender and IBS subgroups in two groups $(\mathrm{P}>0.05)$ (Table 1). 55 patients out of 159 had a history of taking PPI (34.5\%). Also, there was no difference between the consumption rate of PPI among these two groups $(\mathrm{P}=0.61)$.

After three months, one patient from probiotic group and two patients from placebo group have been excluded from the study because they didn't follow the study rules (did not attend the follow ups or not taken medication) (1.8\%). The remaining 156 patients have been examined and HBT has repeated at the end of clinical trial to compare the outcomes.

There was a significant difference between probiotic and placebo groups in terms of their last positive HBT results. Only 8 patients from probiotic group (10.3\%) had positive HBT while 19 patients from placebo group $(24.3 \%)(\mathrm{P}=$ 0.033 ). In addition, the chance of further onset of SIBO was nearly doubled in placebo group in comparison with probiotic group and confidence interval for the frequency of this relation was equal to 0.95 .

The risk of SIBO onset in placebo group compared with probiotic group was $1.831(\mathrm{RR}=$ 1.831 (1.003-3.345)) which demonstrates the higher chance of SIBO onset in placebo group than probiotic group. Therefore, these findings will suggest that probiotics have protective effects on the onset of SIBO and will reduce the chance of SIBO occurrence.

The level of probiotic effects in two groups (all participants) to placebo group were 0.141 $(A R R=0.141)$. Therefore, the chance of successful treatment with probiotics was $14.1 \%$ higher than placebo group. Hence, probiotics have statistically significant effects on reducing the onset of SIBO in patients with IBS (NNT= 7.09). In addition, there wasn't a significant difference in the level of SIBO onset based on IBS subgroups (constipation $7.1 \%$, diarrhea $6.4 \%$, and mixed group 3.8\%) ( $\mathrm{P}=78.0)$. The frequency of SIBO onset in both groups of probiotics and placebo in constipation and mixed type subgroups was not significant $(P>0.05)$. Only in diarrhea type IBS, a statistically significant difference was noticed between probiotic and placebo groups $(\mathrm{P}<0.05)$ (Table 2$)$.

Moreover, the frequency of onset of SIBO in patients with a history of PPI consumption was $5.1 \%$ while in patients with no history of PPI consumption was $12.2 \%(\mathrm{P}=0.65)$. However, this figure did not have significant differences in 
placebo and probiotic groups $(\mathrm{P}>0.50)$ (Table 2). No side-effects were recorded during this clinical trial. Only a few patients reported experiencing bitter mouth feeling while taking probiotics.

Table (1): The comparison between two groups based on gender and IBS subgroups.

\begin{tabular}{|c|c|c|c|}
\hline & Placebo group & Probiotic group & P-value \\
\hline Age average & $36.43 \pm 9.41$ & $36.34 \pm 9.73$ & 0.95 \\
\hline Gender & $27(7 \%)$ & $30(18.9 \%)$ & 0.62 \\
Male & $53(33.3 \%)$ & $49(30.8 \%)$ & \\
Female & & & \\
\hline IBS subgroup & $40(25.2 \%)$ & $36(22.6 \%)$ & 0.48 \\
constipation & $25(15.7 \%)$ & $26(16.4 \%)$ & \\
diarrhea & $15(9.4 \%)$ & $17(10.7 \%)$ & \\
mixed & \multicolumn{2}{|l}{} \\
\hline
\end{tabular}

Table (2): The comparison between the frequency of SIBO onset and consumption of PPI based on gender and IBS subgroups.

\begin{tabular}{|c|c|c|c|c|c|c|}
\hline & & Group & probiotic & Placebo & $\begin{array}{c}\text { Confidence } \\
\text { interval }(95 \% \mathrm{CI}) \\
\end{array}$ & P-value \\
\hline \multirow[t]{4}{*}{ Gender } & \multirow[t]{2}{*}{ Male } & $\begin{array}{c}\text { Negative } \\
\text { HBT }\end{array}$ & $27(48.2 \%)$ & $20(35.7 \%$ & $1.38(0.94-2.02)$ & \multirow[t]{2}{*}{0.27} \\
\hline & & positive HBT & $3(5.4 \%)$ & $6(10.7 \%$ & & \\
\hline & \multirow[t]{2}{*}{ Female } & $\begin{array}{c}\text { Negative } \\
\text { HBT }\end{array}$ & $43(43 \%)$ & $39(39 \%)$ & $1.29(0.74-2.25)$ & \multirow[t]{2}{*}{0.07} \\
\hline & & positive HBT & $5(5 \%)$ & $13(13 \%)$ & & \\
\hline \multirow[t]{6}{*}{$\begin{array}{c}\text { IBS } \\
\text { subgroup }\end{array}$} & \multirow[t]{2}{*}{ Constipation } & $\begin{array}{c}\text { Negative } \\
\text { HBT }\end{array}$ & $32(43.8 \%)$ & $30(41.1 \%)$ & $0.66(0.46-0.97)$ & \multirow[t]{2}{*}{0.19} \\
\hline & & positive HBT & $3(4.1 \%)$ & $8(11 \%)$ & & \\
\hline & \multirow[t]{2}{*}{ Diarrhea } & $\begin{array}{c}\text { Negative } \\
\text { HBT }\end{array}$ & $24(47.1 \%)$ & $17(33.3 \%)$ & $1.25(1.10-1.43)$ & \multirow[t]{2}{*}{0.04} \\
\hline & & positive HBT & $3(3.9 \%)$ & $8(15.7 \%)$ & & \\
\hline & \multirow[t]{2}{*}{ Mixed } & $\begin{array}{c}\text { Negative } \\
\text { HBT }\end{array}$ & $14(43.8 \%)$ & $12(37.5 \%)$ & $0.76(0.39-1.49)$ & \multirow[t]{2}{*}{1.00} \\
\hline & & positive HBT & $3(9.4 \%)$ & $3(9.4 \%)$ & & \\
\hline \multirow[t]{4}{*}{$\begin{array}{l}\text { Medical } \\
\text { history }\end{array}$} & \multirow[t]{2}{*}{$\begin{array}{c}\text { PPI } \\
\text { Consumption }\end{array}$} & $\begin{array}{l}\text { Negative } \\
\text { HBT }\end{array}$ & $24(43.6 \%)$ & $23(41.8 \%)$ & $0.91(0.47-1.78)$ & \multirow[t]{2}{*}{0.06} \\
\hline & & positive HBT & $1(1.8 \%)$ & $7(12.7 \%)$ & & \\
\hline & \multirow[t]{2}{*}{$\begin{array}{l}\text { No PPI } \\
\text { Consumption }\end{array}$} & $\begin{array}{l}\text { Negative } \\
\text { HBT }\end{array}$ & $46(45.5 \%)$ & $36(35.6 \%)$ & $0.48(0.25-0.94)$ & \multirow[t]{2}{*}{2.0} \\
\hline & & $\begin{array}{l}\text { Negative } \\
\text { HBT }\end{array}$ & $7(6.9 \%)$ & $12(11.9 \%)$ & & \\
\hline
\end{tabular}

Masjedizadeh et al., Afro-Egypt J Infect Endem Dis 2020;10(3):279-286

https://aeji.journals.ekb.eg/

http://mis.zu.edu.eg/ajied/home.aspx 


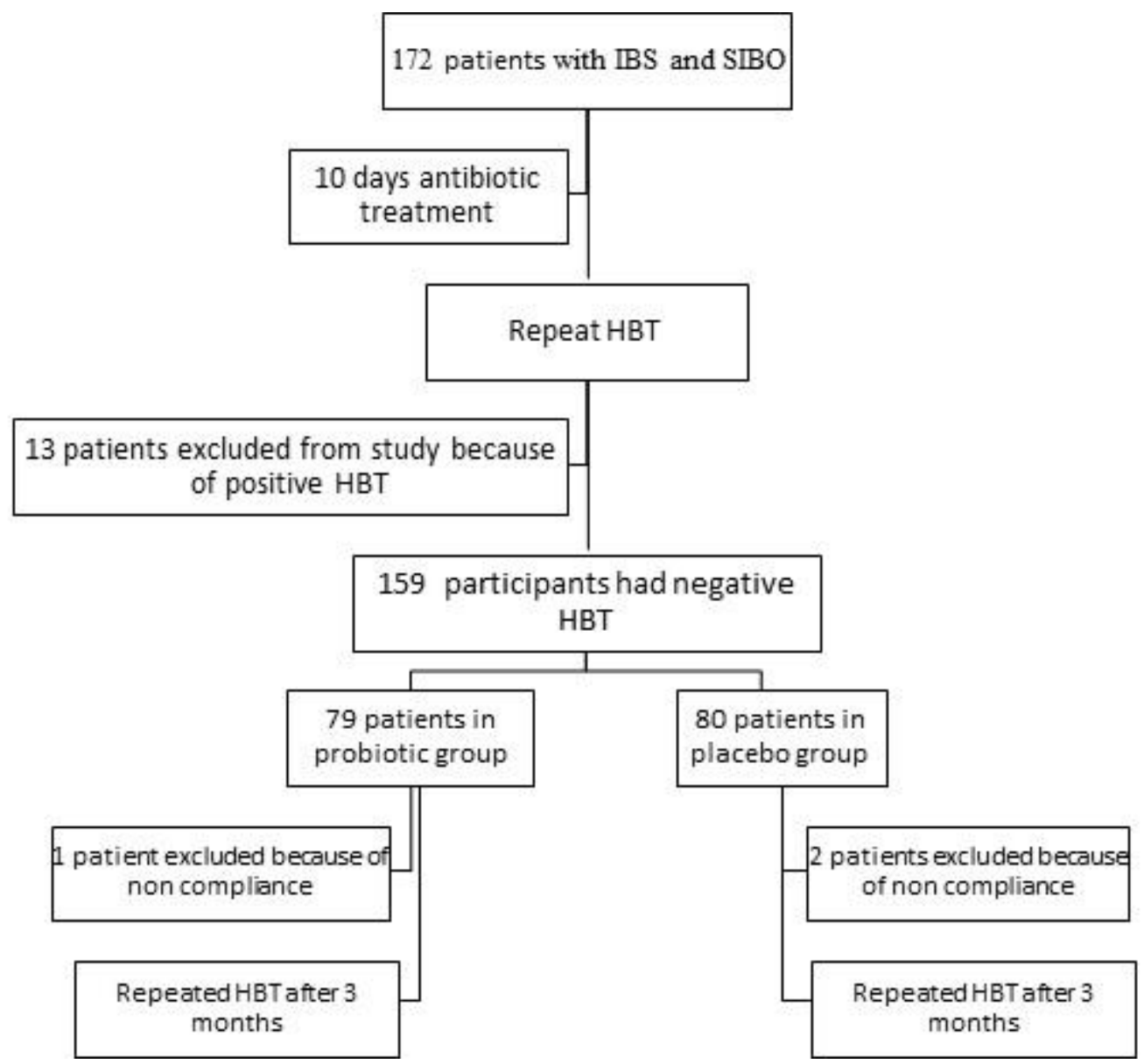

Figure(1): Algorithm of the study

\section{DISCUSSION}

IBS is the most common gastrointestinal disorder and also the most common reason for referring patients to gastrointestinal specialists [6]. Our research findings show that after three months treatment of two different groups with probiotics and placebo, the occurrence of SIBO in probiotic group (Mutaflor containing E.Coli) has significantly decreased in comparison with placebo group.

Despite unclear etiology and no definite treatment, there are some evidences about the role of changes in the normal intestinal bacterial flora and SIBO in IBS pathogenesis. First, differences in pattern of fecal microbes and mucus of IBS patients compared to healthy people. Secondly, increasing evidence regarding the activity of immune system especially lymphocytes and the mast cells in IBS, which shows the existence of infection, process in patient's pathogenesis and the importance of antibiotic treatment in improving IBS patient's symptoms $[1,2,6,10 p]$.
The recent studies suggest that there is a strong link between SIBO and IBS. In some studies, up to 70 to $80 \%$ of IBS patients had a positive HBT (Hydrogen Breath Test) as well as positive glucose and lactose. Therefore, appropriate antibiotic treatment has improved the symptoms of most of the patients $[\mathbf{1}, \mathbf{2}, \mathbf{1 1}, \mathbf{1 2}]$. The above evidence highlights the potential therapeutic effects of modifying digestive system microbes especially with the probiotics for IBS treatment. Although, antibiotics are the main treatment of SIBO, with consideration of reported evidences, antibiotics resistance and recurrence of SIBO after one course of treatment with antibiotics should keep in mind. In this research, we aimed to assess the efficacy of probiotics for prevention of SIBO occurrence after finishing one course of antibiotics treatment to justify the symptoms of IBS patients.

Probiotics are defined as live microorganisms which have beneficial effects on the host's health when introducing in a sufficient doze [1,6]. The exact mechanism of the probiotics function in digestive system health is not clear yet, however,

Masjedizadeh et al., Afro-Egypt J Infect Endem Dis 2020;10(3):279-286 https://aeji.journals.ekb.eg/

http://mis.zu.edu.eg/ajied/home.aspx 
it has been shown that probiotic alone or with antibiotic treatment is stimulating intestinal immune system, improving dysmotility, affecting intestinal oversensitivity, improving fermentation, managing dominant intestinal flora, protecting mucus membrane, improving digestion, preventing bloating, and preventing abdominal dilation and diarrhea among IBS patients $[1,6,9,13,14,15]$.

Although there are many investigations in the past, no study has covered the occurrence of SIBO in IBS patients after antibiotic treatment, as we have aimed in our research. In all research studies regarding the role of probiotics in the treatment of IBS, only the effects of different probiotics in improving IBS symptoms have evaluated with controversial findings. For instance, the effects of Lactobasil plantrom in IBS patients were investigated in a study by Niedzielin (2001, Poland) [16] and in a more recent study by Ducrotte (2011, India) (17). They showed that the severity of symptoms and daily frequency of pain and abdominal bloating had a significantly higher improvement in placebo group. In a similar study by Yu-Jing et al. (2002, China) (1) probiotics including lactobacillusbirobacterium- anticcoc were applied. In a study by Guandalini (2011, USA) [18] probiotic VSL \#3 was used in children with IBS and a research by Sudha (India) [19] used UBO3 capsule. In a study by Gawronska et al. (Poland) [20] Lactobasil GG was used in children. All the above studies showed a significant improvement of abdominal pain, bloating and quality of life of patients with IBS by using probiotics against placebo group.

In addition, a study by Soifer (2010, Argentina) also compared Metronidazole with probiotics consists of lactobacil-streptococ-bifidubacterium in the patients with IBS and SIBO. They showed that probiotics are more effective in improving the symptoms in comparison with antibiotics [21]. On the other hand, another study by Bausserman (2005, USA) [8] used Lactobasil GG and in a study by Eva Niv (2005, Palestine) [22] using Lactobacillus Reuteri as probiotic had no priority over placebo in reduction of abdominal pain and other IBS symptoms. Only abdominal distension and flatulence were slightly different in two groups.

In some studies, the level of SIBO occurrence after a course of treatment with Rifaximin had following outcomes: $12.5 \%$ after 3 months treatment, $27.5 \%$ after 6 months treatment, and 43.7\% after 9 months treatment [5]. However, no research has done so far to assess the long-term effects of probiotics treatment on the SIBO onset especially among IBS patients.

Our research findings also showed that the frequency of SIBO occurrence has no statistically significant difference between two groups. In contrast, a study by Lauritano et al. (2005-2007, Italy) has shown that chronic consumption of PPI had a correlation with higher SIBO occurrence. These findings could explain by the failure of protective effects of stomach acid and more colonization of Oropharyngeal bacterial flora in small intestine [5]. In addition, in our study patient's gender and the types of IBS (constipation and mixed) did not affect the percentage of SIBO. SIBO occurrence was significantly lower in probiotic group compared to placebo group just in diarrhea type of IBS (IBS-D).

The strength of this research is that we applied an objective variable (Hydrogen Breathing Test for assessing SIBO) to examine the effects of probiotics on IBS treatment; while the previous researchers have only studied the effects of probiotics based on subjective variables of IBS symptoms. In addition, in our study more than $98 \%$ of patients have finished the investigation until the end of the research. Furthermore, most of previous studies assess the symptoms of IBS patients after only a short period of (maximum one month) for administrating probiotics, while in our research we have investigated patients after three months.

There are some limitations in this research, for instance, the changes of fecal microbes made by probiotics consist of E.Coli has not been considered in this study. In addition, we did not assess the effects of probiotics on reduction of intestinal gas production and the symptoms of IBS, and the quality of life of patients with IBS. Moreover, further studies with more participants, longer follow up period, using other probiotics or a combination of probiotics recommend in order obtaining more knowledge regarding the efficiency of probiotic organisms in reducing the symptoms of patients with IBS. 


\section{CONCLUSION}

According to the findings of this research, the conclusion is that Mutaflor probiotics containing E.Coli can significantly reduce the onset of SIBO in IBS patients without any side-effects (based on statistical tests). Therefore, this probiotic could be safely used in treatment of IBS.

\section{Acknowledgement}

This study derived from final thesis of Dr Sam Shahinzadeh for his gastroenterology fellowship under supervision of Alimentary Tract Research Center.

\section{Abbreviations}

GI: gastrointestinal; IBS: irritable bowel syndrome; SIBO: small intestine bacterial overgrowth; IBD: inflammatory bowel disease; CRP: C reactive protein; ESR: erythrocyte sedimentation rate; CBC: complete blood count; TTG: tissue Trans glutaminase; HBT: hydrogen breathe test; PPI: proton pomp inhibitor; NNT: number need to treat;

Ethical considerations: This study has been approved by ethical committee of university (ETH-1392) and also has registered in IRCT as 2013122815959N1.

Conflict of interest: The authors have none to declare.

\section{Funding: NO}

\section{REFERENCES}

1. Fan YJ, Chen SJ, Yu YC, Si JM, Liu B. A probiotic treatment containing Lactobacillus, Bifidobacterium and Enterococcus improves IBS symptoms in an open label trial. J Zhejiang Univ Sci B 2006; 7(12): 987-991.

2. Ghoshal UC, Srivastava D, Ghoshal U, Misra A. Breath tests in the diagnosis of small intestinal bacterial overgrowth in patients with irritable bowel syndrome in comparison with quantitative upper gut aspirate culture. Eur J Gastroenterol Hepatol 2014; May 20

3. Pimentel $\mathrm{M}$, Chang $\mathrm{G}$. inflammation and microflora. Gastroenterology Clin North AM 2011; 40(1): 69-85.
4. Hungin APS, Whorwell PJ, Tack J, Mearins F. The prevalence, patterns and impact of irritable bowel syndrome: an international survey of 40000 subjects. Aliment Pharmacol Ther 2003; 17: 643-650.

5. Sachdeva S, Rawat AK, Reddy RS, Puri AS. Small intestinal bacterial overgrowth (SIBO) in irritable bowel syndrome: Frequency and predictors. J Gastroenterology and Hepatology 2011; 26(3): 135-138

6. Lauritano EC, Gabrielli M, Scarpellini E, Lupascu A, Novi M, Sottili S et al. Small Intestinal Bacterial Overgrowth Recurrence After Antibiotic Therapy. Am J Gastroenterol 2008; 103(8): 2031-2035.

7. Parkes GC, Sanderson JD, Whelan K. Symposium on 'Dietary management of disease' Treating irritable bowel syndrom with probiotics: the evidence. Proceeding of the Nutrition Society .2010; 69:187-194.

8. Bausserman $M$, Michail S. The use of lactobacillus GG in irritable bowel syndrome in children : a double-bilnd randomized control trial. The Journal of Pediatrics 2005; 147:197201.

9. Whelan K. Probiotics and prebiotics in the management of irritable bowel syndrome: a review of recent clinical trials and systematic reviews. Curr Opin Clin Nutr Metab Care. 2011; 14(6): 581-587.

10. Owyang C. Irritable Bowel syndrome. In: Longo DL, Kasper DL, Jameson JL, et al. Harrison's principles of internal medicine. 18th. New York: MC Graw Hill; 2012:2469-2501.

11. Koo HL, Sabounchi S, Huang DB, DuPont HL. Rifaximin Therapy of Irritable Bowel Syndrome. Clinical Medicine Insights: Gastroenterology 2012; 5: 31-41.

12. Simren M, Stotzer PO. Use and abuse of hydrogen breath tests. Gut 2006; 55: 297-303.

13. Verdu EF, Collins SM. Irritable bowel syndrome and probiotics: from rationale to clinical use. Curr Opin Gastroenterol 2005; 21: 697-701.

14. Rolfe RD. Symposium: Probiotic Bacteria: Implications for Human Health, The Role of Probiotic Cultures in the Control of Gastrointestinal Health. J Nutr 2000; 130: 396S$402 \mathrm{~S}$. 
15. Barrett JS, Canale KEK, Gearry RB, Irving PM, Gibson PR. . Probiotic effects on intestinal fermentation patterns in patients with irritable bowel syndrome. World J Gastroenterol 2008; 14(32): 5020-5024.

16. Niedzielin K, Kordecki H, Birkenfeld B. A controlled, double-blind, randomized study on the efficacy of lactobacillus plantaum $299 \mathrm{~V}$ in patients with irritable bowel syndrome. European J Gastroenterology and Hepatology 2001; 13(10):1143-1147.

17. Ducrotté P, Sawant P, Jayanthi V. Clinical trial: Lactobacillus plantarum 299v (DSM 9843) improves symptoms of irritable bowel syndrome. World J Gastroenterol 2012; 18(30): 4012-4018.

18. Guandalini S, Magazzù G, Chiaro A, La Balestra V, Di Nardo G, Gopalan Set al. VSL\#3 improves symptoms in children with irritable bowel syndrome: a multicenter, randomized, placebocontrolled, double-blind, crossover study. $J$ Pediatr Gastroenterol Nutr 2010 Jul; 51(1): 2430.

19. Sudha MR, Sawant P. Effect of capsule 'UB03' containing potential probiotic strains for the treatment of patients with irritable bowel syndrome. Benef Microbes 2011; 2(3):229-33.
20. Gawronska A, Dziechciarz P, Horvath A, Szajewska H. A randomized double-blind placebo-controlled trial of Lactobacillus GG for abdominal pain disorders in children. Aliment Pharmacol Ther 2007; 25: 177-184.

21. Soifer LO, Peralta D, Dima G, Besasso H. Comparative clinical efficacy of a probiotic vs. an antibiotic in the treatment of patients with intestinal bacterial overgrowth and chronic abdominal functional distension: a pilot study. Acta Gastroenterol Latinoam 2010;40(4):323-7

22. Niv E, Naftali T, Hallak R, Vaisman N. The efficacy of Lactobacillus reuteri ATCC 55730 in the treatment of patients with irritable bowel syndrome - a double blind, placebo-controlled, randomized study. Clinical Nutrition 2005; 24 : 925-931.

23. Palsson OS, Whitehead WE, Van Tilburg MA, Chang L, Chey W, Crowell MD, et al. Development and validation of the Rome IV diagnostic questionnaire for adults. Gastroenterology 2016; 150(6):1481-91. 